\title{
Time-domain behavior of blazar OJ 287 and the binary supermassive black hole conjecture
}

\author{
Stefano Ciprini* \\ Space Science Data Center ASI, Rome I-00133, Italy \\ INFN Section of Perugia, Perugia I-06123, Italy \\ E-mail: stefano.cipriniessdc.asi.it
}

\section{Mauri J. Valtonen}

Finnish Centre for Astronomy with ESO, University of Turku, Turku FIN-21500, Finland

Tuorla Observatory, Dept. of Physics and Astronomy, Univ. of Turku, Turku FIN-21500, Finland

\section{Staszek Zola}

Astronomical Observatory, Jagiellonian University, Krakow PL-30-244, Poland

Mt. Suhora Observatory, Pedagogical University, Krakow PL-30-084, Poland

\section{Arti Goyal}

Astronomical Observatory, Jagiellonian University, Krakow PL-30-244, Poland

\section{Pauli Pihajoki}

University of Helsinki, Department of Physics, Helsinki FIN-00560, Finland

\begin{abstract}
The proper understanding of blazar variability at the various electromagnetic spectral bands is one goal of multifrequency astrophysics. In this frame a peculiar and controversial phenomenology is the periodicity, postulated for long-term radio or optical flux light curves of about a dozen of blazars. The well-known BL Lac object OJ 287 (PKS 0851+202, S3 0851+20, PG 0851+202, $z=0.306$ ) is not only a high-variable, peculiar, extragalactic source with hints for approximatively cyclical optical outbursts, but it also represents a case of substantial intensive and extensive (longterm) multifrequency observations. This rich database allow us a deeper analysis based on a wide range of variability timescales with some recent results that are highlighted here.
\end{abstract}

7th Fermi Symposium 2017

15-20 October 2017

Garmisch-Partenkirchen, Germany

\footnotetext{
* Speaker.
} 


\section{130-year observations of the blazar OJ 287}

Periodicity in extragalactic objects (galaxies, AGN) is a peculiar and controversial phenomenology claimed, for example, for radio/optical flux light curves of some blazars. Estimates of periodicity significance, the red-noise estimation, gaps in light curves and systematics, generally makes the results weaker for blazar/AGN sources with respect to star-sized systems like the X-ray binaries in our Galaxy and their quasi-periodic oscillations. One of the most cited case is blazar OJ 287 (also known as PKS 0851+202, S3 0851+20, B2 0852+20, PG 0851+202 in literature, and as 1ES 0851+203, RX J0854.8+2006, 3EG J0853+1941, 3FGL J0854.8+2006 in high-energy bands). OJ 287 (Fig. 1) is an intermediate synchrotron-energy peaked and optically high-variable BL Lac object (variations $>3 \mathrm{mag}$ ) placed at $z=0.306$, having one of the largest database of radio-/opticalmonitor data (optical plates dates back to year 1888, see [10,3] for a review of earlier data) and representing one of known cases in literature for an extragalactic source with periodicity claims.

Coordinated, both intensive and long-term, observing programs devoted to OJ 287 were organized and accomplished in the past years: the 1993-1997 OJ-94 monitoring project ${ }^{1}$; the 2005-2008 ENIGMA and WEBT projects with the related long-term and intensive multifrequency observing campaigns $^{2}$; the 2006-2017 optical long-term monitoring project ${ }^{3}$.

The postulated cyclical temporal modulation of the optical flux emitted by OJ 287 has been interpreted, for example, by a precessing jet, a rotating helical jet, or a binary super-massive black hole (SMBH) system with sub-parsec separation and high-mass ratio $(100: 1)[9,11,14]$. The presence of a binary SMBH system with sub-parsec separation in OJ 287 is based on the approximatively periodical optical flares observed and arising, in this hypothesis, from the secondary $\mathrm{BH}$ perturbing or piercing (Fig. 1) the accretion disk of the primary, about every 11-12 years, with nine
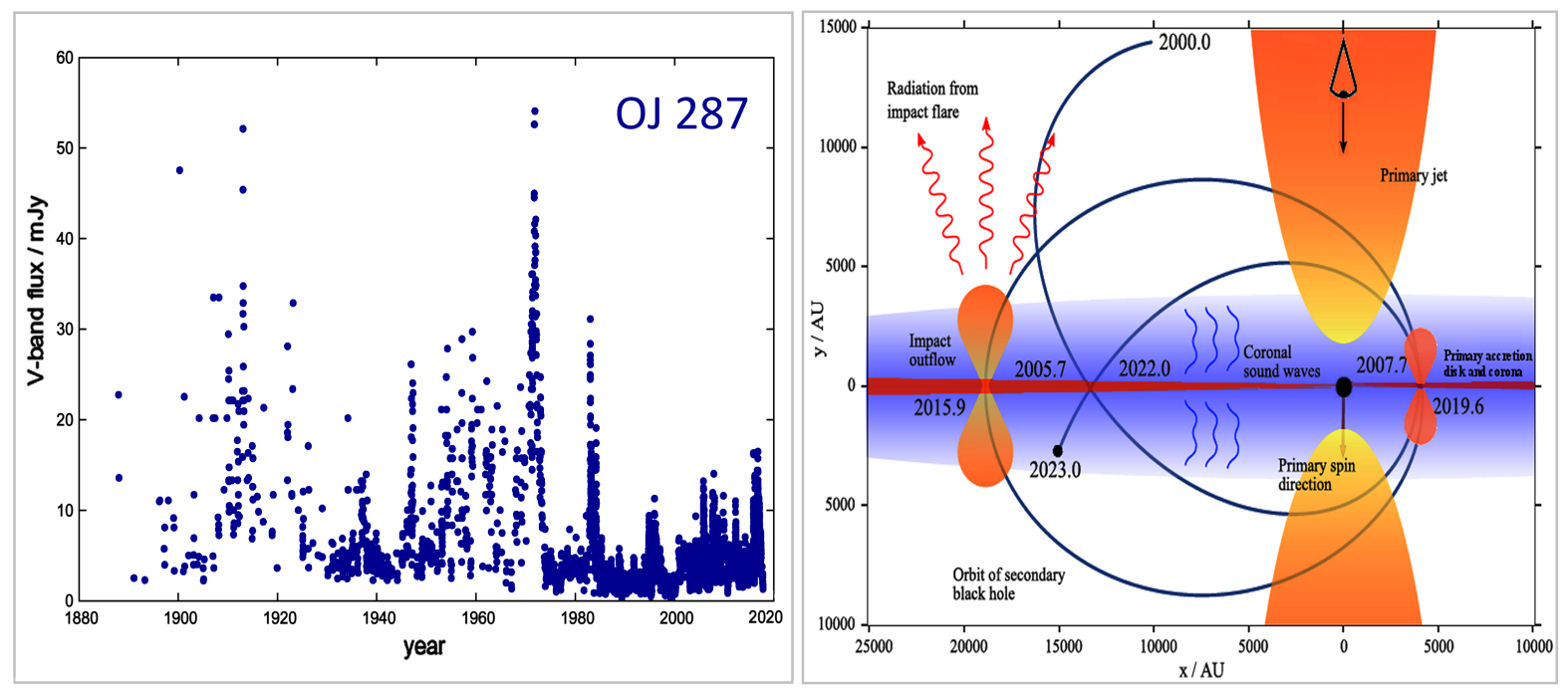

Figure 1: Left panel: about 130-year optical light curve of OJ 287. Right panel: Pictorial representation of the orbit of the secondary SMBH in OJ 287 from 2000 to 2023. Optical outbursts are seen every time the secondary SMBH impacts/perturbs the accretion disk of the primary SMBH (adapted from [14]).

\footnotetext{
${ }^{1}$ http://www.astro.utu.fi/research/oj94/

${ }^{2}$ http: //www.astro.utu.fi/OJ287MMVI/

${ }^{3}$ http://www.as.up.krakow.pl/sz/oj287.html
} 
identified episodes since 1913. Under this hypothesis the General Relativity orbital precession of the secondary BH naturally explains the quasi-periodic optical oscillations of OJ 287 [11, 14].

\section{Some recent results}

The last two, and best monitored, optical outbursts phases of OJ 287 occurred at the end of 2005, (with a secondary event in 2007), and in the period between winter 2015 and summer 2016. The optical monitor and multifrequency data obtained with coordinated observing campaigns ${ }^{2}$ performed in the years between 2005 and 2008 [1] allowed, in particular, to test the Einstein's General Relativity (GR) theory in strong-field conditions [11] through light curve timing (clocking). Such data also corroborated the sub-parsec binary SMBH hypothesis, where optical/UV/X-ray data have evidenced the separation between un-boosted spectral component dominated by thermal bremsstrahlung emission from hot $\left(3 \times 10^{5}{ }^{\circ} \mathrm{K}\right)$ gas and the relativistically beamed in-jet, nonthermal, X-ray emission. The results where obtained thanks to XMM-Newton EPIC and OM observations [1, 13, 12], and to Swift XRT and UVOT data [14] based on Guest Observer programs and several $\mathrm{ToO}$ proposals. The dedicated Swift time-domain experiment is continuing since winter 2015 trough 2016 and 2017 thanks to several observing proposals and ToOs. Fig. 2 (left panel) shows the different temporal shape between UV and X-ray data during the impact bremsstrahlung outburst of Nov.-Dec. 2015. Other observing programs confirmed the thermal spectral bump dominating from UV to near-IR bands during this epoch [4], in agreement also with optical polarization data showing a low-polarization (thermal) component during the impact epoch [16, 14].

From the earlier 11/12-year optical periodicity claims, the current theory for OJ 287 points to a more complex, non-strictly periodic, scenario, with double outburst events (separated by one/two years) and orbital precession and shrinking as clear signatures in the light curves [11, 13, 14]. GR three-body calculations and simulations demonstrates that a SMBH surrounded by a gas disk (the third body as an ensemble of particles) and possessing a companion SMBH can create a quasiperiodic signal. From the light curve timing/clocking is possible to determine the secondary orbit and the sub-parsec binary SMBH system parameters. The secondary passes through the accretion disk of the primary with Keplerian orbit and post-Newtonian GR corrections at the third order expansion. Specifically post-Newtonian equations of motion for compact SMBH inspiralling binaries with high-mass ratio are adopted, with Lagrangian and Hamiltonian formalisms, and numerical computations of the gravitational reaction "self-force". The loss in gravitational binding energy is caused by low frequency gravitational wave $(\mathrm{GW})$ emission and the Lense-Thirring effect: the binary orbital plane precess, mainly due to the spin of the primary SMBH [11, 14]. In high-mass ratio binaries the perturbations around the primary Kerr SMBH and the orbit decay of the secondary due to GW radiation, turned out to be a challenging (formalism and computational) problem: a consistent formalism for the "self-force" corrections to the background geodesic motion are available only since the end of ' 90 s $[8,6]$. At the impact of the secondary on the accretion disk, a hot bubble of gas is extracted from the disk and expands at its internal sound speed. When it becomes optically thin during the expansion a burst of optical-UV radiation occurs.

The timescale from two galaxy merger to their central SMBH merger is usually of the order of $10^{8}-10^{9}$ years, and at the OJ 287 sub-parsec scale the time to merge is $<10^{5}$ years. The estimated parameters of the system are: SMBHs masses $m_{1}=(1.84 \pm 0.01) \times 10^{10} \mathrm{M}_{\odot} ; m_{2}=$ 

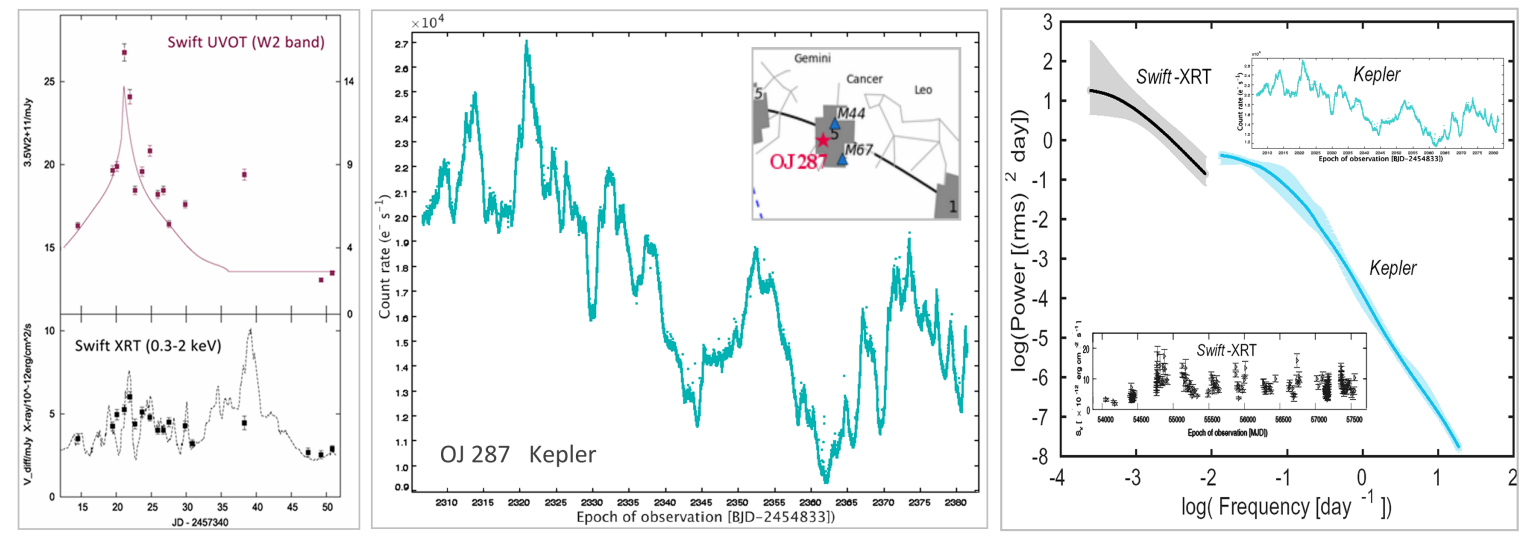

Figure 2: Left panel: Swift XRT X-ray and UVOT UV light curves during the predicted impact outburst epoch for OJ 287 of Nov.-Dec. 2015. The model foreseen the separation of the disk bremsstrahlung (optical and UV flux) from erratic in-jet synchrotron/inverse-Compton beamed variability (X-ray flux). The orphan optical-UV outburst strengthened the evidence for an extra, optical-UV, out of the jet, emission (adapted from [14]). Central panel: the 1-minute sampling optical light curve from Apr. 27 to Jul. 13, 2015 of OJ 287 obtained by the Kepler space telescope (K2 mission, Campaign \#5). Right panel: temporal variability power density spectrum of OJ 287 constructed with Kepler and Swift data (adapted from [2]).

$(1.40 \pm 0.03) \times 10^{8} \mathrm{M}_{\odot} ; \Delta \phi=(39.1 \pm 0.1)^{\circ} ;$ primary Kerr SMBH spin $\chi=(0.313 \pm 0.01) ; e=$ $0.66 \pm 0.01 ; q=1.0 \pm 0.8[12,13,14]$. In particular the orbital motion is measurably different if the primary BH has no "hair" (no-hair theorem of GR) or if it has some "hair". The external gravitational field depends strictly only on the mass and the spin (net electric charge not expected in astronomical BHs). The occurrence of the optical outburst within the expected time window, using the high-mass ratio compact binary model is consistent with the no-hair theorem at the second post-Newtonian order, and confirms the energy losses by GW radiation in agreement within a few percents with the prediction by GR. The binary modeling of OJ 287 also indicated that the primary Kerr SMBH should spin approximately at quarter of the maximum spin rate allowed in GR, with a unique mathematical solution and prediction for impact flare outburst, that was observed on Dec. 2015. Its clocking is spin-sensitive, and this accurate light curve timing allowed us to constrain the Kerr spin parameter of the primary $\chi=(0.313 \pm 0.01)$ [14]. The primary SMBH in OJ 287 is a BH described by General Relativity with $30 \%$ accuracy and the outburst clocking also confirmed the energy loss by very low-frequency GW emission.

In summary OJ 287 can represent an optimal laboratory for GR in strong field conditions (masses, orbital parameters, no-hair theorem, precession, GW radiation losses). On the other hand the big effort in temporal time-domain monitor and multi-frequency observations allows us, in general, to perform several studies about the spectral and temporal behavior of this blazar, on both short and long time scales, and during different brightness states.

The search for different physical mechanisms responsible for the variability is not necessarily related only to the binary system underlying, deterministic, hypothesis. High-energy observations at X-ray bands (BeppoSAX, XMM-Newton, Suzaku, Swift, $[5,1,14]$ ) and gamma-ray bands (Fermi all-sky survey and continuous monitoring since Aug. 2008) are now contributing to enlarge the physical picture on this peculiar extragalactic source, and are contributing to increase the power of synergetic multifrequency analysis.

Optical flux light curves during the 2015-2016 observing season where also recently analyzed 
searching for quasi-periodic oscillations (QPOs) corresponding to the innermost stable circular orbit (ISCO) timescale of the primary SMBH [16]. Previously, the long-term (2005-2008) observing campaign data evidenced the possible existence of a $\sim 50$-day quasi periodic primary ISCO [7]. The optical flux and polarization data of the following 2016-2017 observing season indicated a persisting activity with high levels of polarization degree indicating a synchrotron emission from the primary SMBH jet, explained as in-jet flares induced by accretion perturbations following the Nov. 2015 impact [15].

An intensive and continuous three-month observing campaign of OJ 287 was performed by the Kepler space telescope, with 1 minute sampling at $>90 \%$ duty cycle and high $\mathrm{S} / \mathrm{N}$, during the K2 Campaign \#5 (Apr.27-Jul.13 2015, [16, 2] and Fig. 2, central panel). No statistically significant QPO was detected in the range from minutes to 30 days.

The 77-day continuous Kepler light curve and the gapped 2015-2017 Swift light curve, allowed us to determine the temporal variability power density spectrum (PDS) of OJ 287 on more than four decades ([2] and Fig. 2 right panel). The overall optical PDS calculated with Swift-UVOT and Kepler data is mostly consistent with, stochastic, red noise process, no QPOs and a hint for a mild break at 10-day timescale. The X-ray (Swift-XRT) PDS is a pure red noise description [2].

There is not a strict theoretical upper limit to the mass grow of a black hole, it depends on its age and how fast is the accretion. The primary SMBH of OJ 287 is among the most massive known black hole in the universe $\left(m_{1}=(1.84 \pm 0.01) \times 10^{10} \mathrm{M}_{\odot}\right)$. OJ 287 could have provided the first indirect evidence for the existence of a compact binary supermassive spinning black hole system emitting gravitational waves and, possibly, constituting an indirect test bench for General Relativity in strong field. This is encouraging for the Pulsar Timing Array efforts that will directly detect, in the next future, very low frequency gravitational waves $\left(10^{-9}-10^{-7} \mathrm{~Hz}\right)$ from such binary SMBHs in the early stages of inspiral. More tests for the binary hypothesis in OJ 287 will be possible in 2019 based on the next predicted outburst event.

\section{References}

[1] S. Ciprini, C. M. Raiteri, N. Rizzi, I. Agudo, et al. 2007, Prominent activity of the blazar OJ 287 in 2005. XMM-Newton and multiwavelength observations, Mem. SAIt, 78, 741

[2] A. Goyal, L. Stawarz, S. Zola, V. Marchenko, et al. 2017, Stochastic modeling of multiwavelength variability of the classical BL Lac object OJ 287 on timescales ranging from decades to hours, ApJ, submitted, [arXiv:1709.04457]

[3] R. Hudec, M. Bašta, P. Pihajoki, \& Valtonen M. 2013, The historical 1900 and 1913 outbursts of the binary blazar candidate OJ 287, A\&A, 559, A20

[4] P. Kushwaha, A. C. Gupta, P. J. Wiita, H. Gaur, et al. 2018, Multiwavelength temporal and spectral variability of the blazar OJ 287 during and after the 2015 December flare: a major accretion disc contribution, MNRAS, 473, 1145, [arXiv:1709.04957]

[5] E. Massaro, P. Giommi, M. Perri, G. Tagliaferri, et al. 2003, Optical and X-ray observations of the two BL Lac objects OJ 287 and $M S 1458+22$, A\&A, 399, 33

[6] H. Nakano, Y. Zlochower, C. O. Lousto, \& M. Campanelli 2011, Intermediate-mass-ratio black hole binaries. II. Modeling trajectories and gravitational waveforms, Phys. Rev. D, 84, 124006. [arXiv: 1108.4421 ]

[7] P. Pihajoki, M. Valtonen \& S. Ciprini 2013, Short time-scale periodicity in OJ 287, MNRAS, 434, 3122, [arXiv:1307.1113] 
[8] T. C. Quinn \& R. M. Wald, 1997, Axiomatic approach to electromagnetic and gravitational radiation reaction of particles in curved spacetime, Phys. Rev. D, 56, 3381, [arXiv: gr-qc/9610053]

[9] A. Sillanpää, S. Haarala, M. J. Valtonen, B. Sundelius \& G. G. Byrd 1988, OJ 287 - Binary pair of supermassive black holes, ApJ, 325, 628

[10] L. O. Takalo 1994, OJ 287: The Rosetta stone of blazars, Vistas in Astron., 38, 77

[11] M. J. Valtonen, H. J. Lehto, K. Nilsson, J. Heidt, et al. 2008, A massive binary black-hole system in OJ287 and a test of general relativity, Nature, 452, 851, [arXiv : 0809.1280]

[12] M. Valtonen \& S. Ciprini 2012, OJ 287 binary black hole system, Mem. SAIt, 83, 219, [arXiv: 1112 .1162]

[13] M. J. Valtonen, S. Ciprini \& H. J. Lehto 2012, On the masses of OJ287 black holes, MNRAS, 427, 77, [arXiv:1208.0906]

[14] M. J. Valtonen, S. Zola, S. Ciprini, A. Gopakumar et al. 2016, Primary Black Hole Spin in OJ 287 as Determined by the General Relativity Centenary Flare, ApJ Lett., 819, L37, [arXiv: 0809.1280]

[15] M. Valtonen, S. Zola, H. Jermak, S. Ciprini et al. 2017, Polarization and Spectral Energy Distribution in OJ 287 during the 2016/17 Outbursts, Galaxies, 5, 83

[16] S. Zola, M. Valtonen, G. Bhatta, A. Goyal, et al. 2016, A Search for QPOs in the Blazar OJ287: Preliminary Results from the 2015/2016 Observing Campaign, Galaxies, 4, 41 